\title{
A reforma da Atenção Primária à Saúde e a utilização dos serviços ambulatoriais no município do Rio de Janeiro (RJ), Brasil
}

The reform of Primary Health Care and the use of outpatient services in the municipality of Rio de Janeiro (RJ), Brazil

RESUMO O município do Rio de Janeiro realizou, a partir de 2009, uma reforma do setor saúde, com foco na Atenção Primária à Saúde (APS). O artigo tem por objetivo analisar a capacidade de governo do município do Rio de Janeiro sobre procedimentos ambulatoriais comuns e a sua retenção em estabelecimentos orientados pela APS, considerando a utilização dos serviços ambulatoriais ao final do primeiro ciclo administrativo da reforma. Os dados sugerem efeitos não intencionais da política, onde a ênfase na universalização da APS reforçou a atuação do município na atenção ambulatorial e, consequentemente, na especialização de funções federativas na direção dos preceitos da Reforma Sanitária Brasileira.

PALAVRAS-CHAVE Atenção Primária à Saúde. Assistência ambulatorial. Avaliação em saúde.

ABSTRACT The municipality of Rio de Janeiro accomplished, from 2009, a reform of the health sector, focusing on Primary Health Care (PHC). This article aims to analyze the capacity of government of the municipality of Rio de Janeiro on common outpatient procedures and its retention in establishments guided by $\mathrm{PHC}$, considering the use of outpatient services at the end of the first administrative reform cycle. The data suggest unintended effects of the policy, where the emphasis on the universalization of PHC strengthened the role of the municipality in outpatient care and, therefore, in the specialization of federal functions towards the precepts of the Brazilian Health Reform.

KEYWORDS Primary Health Care. Ambulatory care. Health evaluation.

1 Universidade do Estado do Rio de Janeiro (Uerj), Instituto de Medicina Social (IMS) - Rio de Janeiro (RJ), Brasil. gzportela.fiocruz@gmail. com

2 Fundação Oswaldo Cruz (Fiocruz), Escola Nacional de Saúde Pública Sergio Arouca (Ensp) - Rio de Janeiro (RJ), Brasil.

ribeiro@ensp.fiocruz.br 


\section{Introdução}

O município do Rio de Janeiro realizou, a partir de 2009, uma reforma do setor saúde, com foco nos serviços de Atenção Primária à Saúde (APS) e na adoção das Organizações Sociais (OS) como modelo de parceria para a sua gestão (RIBEIRO; ALCOFORADO, 2016).

A APS usualmente é compreendida pela definição clássica de Starfield (2002) e representa o primeiro contato dentro do sistema de saúde (o acesso), que, somado à longitudinalidade, à integralidade e à coordenação, conformam os atributos essenciais desse nível de atenção.

Todavia, ainda que o termo seja comumente utilizado de forma abrangente, referindo-se a uma concepção de modelo assistencial e de organização dos sistemas de saúde, ele ainda apresenta pelo menos duas outras interpretações principais: i) programa seletivo, com cesta restrita de serviços; ii) nível de atenção correspondente aos serviços ambulatoriais não especializados de primeiro contato, ou seja, de entrada de pessoas nos sistemas de saúde (GIOVANELLA, 2006; GIOVANELLA, 2011).

$\mathrm{Na}$ abordagem seletiva, geralmente, as decisões a respeito do conteúdo dos benefícios são determinadas por considerações sobre custos. Em grande parte dos países periféricos, com esquema de proteção social de caráter residual, a cobertura da APS é considerada segmentada para o controle de determinados agravos, pouco integral e com importantes desigualdades no acesso (CUETO, 2004; GIOVANELLA, 2008).

$\mathrm{Na}$ outra perspectiva, alguns estudos, principalmente de origem internacional, referem-se à APS como a atenção ambulatorial de primeiro nível, direcionada a resolver a maioria dos problemas de saúde de uma população (GIOVANELLA, 2006; GIOVANELLA, 2011; WHO, 1996). Tradicionalmente, essa abordagem está mais presente em países europeus com sistemas universais públicos, associada à disponibilidade de médicos generalistas (com especialização em medicina geral ou medicina de família e comunidade).

A associação entre a APS e os serviços ambulatoriais também pode ser verificada em outros países. Nos Estados Unidos, por exemplo, uma das principais fontes de informações a respeito da natureza da atenção primária é a Nacional Ambulatory Medical Care Survey (Pesquisa Nacional de Atenção Médica Ambulatorial) (STARFIELD, 2002). Também nos Estados Unidos, como na Europa, abordagens de case-mix, que combinam os diagnósticos em um número gerenciável de categorias diferentes, têm sido aplicadas para descrever a APS, a exemplo do sistema ACG Adjusted Clinical Groups (Grupos Clínicos Ajustados), antigo Ambulatory Care Groups (Grupo de Atenção Ambulatorial) (STARFIELD, 2002).

Conceitualmente, a assistência ambulatorial corresponde aos cuidados ou tratamentos prestados aos indivíduos cujas condições de saúde lhes permitem comparecer à consulta e retornar à casa, dispensando internação hospitalar (OECD; WHO; EUROSTAT, 2011). Ou seja, onde a maioria das interações entre as pessoas e os serviços de saúde ocorre.

Nesse sentido, Berman (2000) demonstra que, ainda que exista diferença de concepção com relação à APS, a atenção ambulatorial pode ser incorporada ao conjunto de atividades consideradas básicas para efeito de avaliação de desempenho dos sistemas nacionais de saúde. Segundo o autor, em países de baixa renda, a maior parte da população se dirige a esses serviços ambulatoriais para a prevenção ou tratamento da maioria das doenças comuns, incluindo diarreia infantil, infecções respiratórias comuns, tuberculose, doenças sexualmente transmissíveis e malária aguda.

Dada a convergência dos conceitos, o estudo tem como hipótese central a de que 
a reforma do setor saúde ocorrida no Rio de Janeiro, com foco na APS, tenha contribuído para a separação de funções federativas no tocante à prestação de serviços usuais no manejo dos usuários no ambiente ambulatorial, ou seja, o município fortaleceu o seu comando sobre os serviços de saúde que realizam procedimentos dessa natureza.

Reforça essa suposição o estudo de Portela (2013), que, ao analisar a utilização e os aspectos centrais do financiamento dos serviços de APS no Rio de Janeiro, ao final do primeiro ciclo administrativo da reforma, apresenta evidências de que ocorreu de fato um processo de especialização na provisão de serviços de APS e de que, em 2012, o município realizava procedimentos ambulatoriais em alta escala.

A segunda hipótese é a de que a maioria desses procedimentos esteja concentrada em estabelecimentos tipicamente ambulatoriais, que incluem as clínicas da família, que devem ser responsáveis por atender às necessidades mais comuns da população.

Dessa forma, este trabalho analisa a capacidade de governo do município do Rio de Janeiro sobre procedimentos ambulatoriais comuns e sua retenção em estabelecimentos orientados pela APS, considerando a utilização dos serviços ambulatoriais ao final do primeiro ciclo administrativo da reforma.

\section{Reforma da Atenção Primária à Saúde no município do Rio de Janeiro}

O município do Rio de Janeiro promoveu uma reforma no setor saúde com ênfase na universalização da APS e na adoção da Estratégia Saúde da Família (ESF) como modelo de reorganização dos serviços.

Essa realidade pôde ser capturada ao se analisar dados referentes ao município no período correspondente ao primeiro ciclo administrativo da reforma (2009-2012), através de pesquisa em Sistemas de Informação em Saúde do Ministério da Saúde.

Após consulta ao Sistema de Informações sobre Orçamentos Públicos em Saúde (Siops/ MS) e comparação dos percentuais mínimos da receita do município, definidos pela Emenda Constitucional no 29/00 (EC-29), a serem vinculados ao setor saúde com aqueles de fato aplicados, percebe-se que no Rio de Janeiro a meta foi ultrapassada em todo o período, com destaque para os três últimos anos da série, que se encerra com $23,5 \%$ da receita própria do município aplicados em saúde. Esse comportamento é observado no gráfico 1, que apresenta a evolução do percentual da receita vinculado à saúde comparada à estipulada pela EC-29 no período de dez anos (2002-2012), que antecederam o fim do primeiro ciclo da administração municipal que implementou a reforma. 
Gráfico 1. Evolução do percentual mínimo da receita vinculado à saúde de acordo com a EC-29 e do aplicado pelo município do Rio de Janeiro. 2002/2012

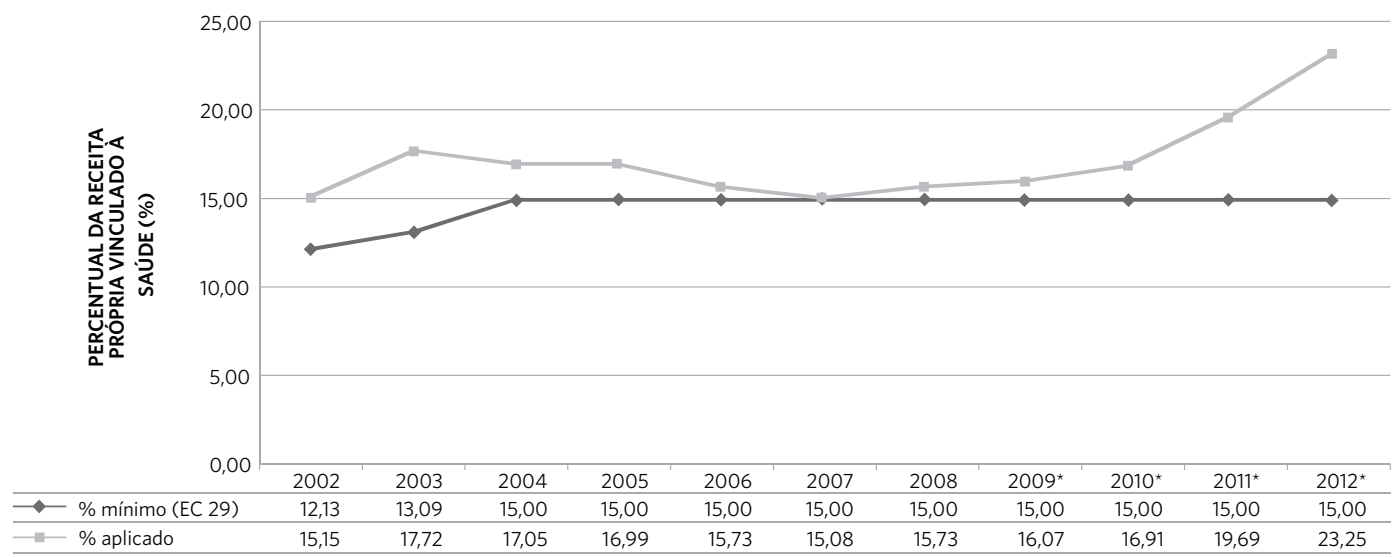

Fonte: Elaboração própria. Siops/MS.

* Período equivalente ao primeiro ciclo administrativo da reforma municipal do setor saúde (2009-2012).

Houve tendência geral de aumento do percentual da despesa liquidada pelo município com APS sobre o total da despesa liquidada com o setor saúde, que, em 2009, foi de $4,9 \%$ (R\$ 247.810.960,49), e, em 2012, de $31,1 \%$, ultrapassando a ordem de um bilhão de reais $(\mathrm{R} \$ 1.158 .946 .909,63)$.

A evolução do percentual médio de cobertura da ESF também revelou abrupta elevação ao longo do período, que se inicia em 2009 , com $11,5 \%$ da população residente cobertos (713.737 pessoas cadastradas), e se encerra em 2012, com 31,0\% (1.982.718 pessoas cadastradas). É importante ressaltar que os dados refletem o aumento da cobertura dos estabelecimentos qualificados como Unidades/Equipes de Saúde da Família, mas não estimam o total dos novos serviços criados, pois parte do conjunto já existia e foi apenas convertida para a ESF.

A adesão do município ao modelo preconizado pelo MS é condizente com o próprio apoio do governo federal ao governo municipal do Rio de Janeiro, costurado à época no plano nacional como parte da aliança, considerada prioritária pelo Planalto, entre o Partido dos Trabalhadores (PT) e o Partido do Movimento Democrático Brasileiro (PMDB).

Essa administração também foi caracterizada pela opção política da Secretaria Municipal de Saúde (SMS) de firmar parceria com OS, mediante a celebração de Contrato de Gestão, na prestação de assistência à saúde da população no âmbito das suas Áreas de Planejamento (AP).

Depois do advento do marco regulatório federal das OS, várias leis cuidaram do tema em outros entes da federação. No âmbito do município do Rio de Janeiro, a SMS publicou, a partir de 2009, uma série de editais de convocação pública para parcerias com entidades qualificadas como tal. Em uma abordagem mais detalhada, Ribeiro e Alcoforado (2016) analisam o desenho institucional da SMS após as reformas adotadas em direção a esse modelo e a capacidade regulatória e a sustentabilidade das escolhas governamentais. 


\section{Procedimentos metodológicos}

Realizou-se um estudo seccional de caráter analítico para o ano de 2012 sobre a utilização de serviços ambulatoriais no Rio de Janeiro, para a prevenção ou tratamento da maioria das doenças comuns.

Em revisão bibliográfica realizada por Travassos e Martins (2004), o conceito de utilização é distinguido do conceito de acesso. As autoras alertam que cada um desses processos corresponde a um modelo explicativo distinto. Enquanto o acesso é uma dimensão do desempenho dos sistemas de saúde associada à oferta, a utilização dos serviços de saúde representa o centro do funcionamento dos sistemas de saúde e compreende todo contato direto ou indireto com os serviços.

Inicialmente, fizeram parte do estudo todos os serviços de saúde localizados no município do Rio de Janeiro (total de 384) que registraram, segundo o SIA-SUS (Sistema de Informações Ambulatoriais do Sistema Único de Saúde), alguma atividade ambulatorial no ano de 2012, ou seja, foi desenhado em caráter censitário e analisou toda a produção de procedimentos ambulatoriais selecionados como indicadores da utilização.

Os serviços foram categorizados e reagrupados segundo informações presentes no Cadastro Nacional de Estabelecimentos de Saúde (CNES), de acordo com as semelhanças julgadas características de tipos distintos de estrutura e do atendimento prestado, em: 'estabelecimento ambulatorial' (centro de atenção psicossocial, centro de saúde/ unidade básica, clínica/centro de especialidade, policlínica, posto de saúde); 'serviço de apoio diagnóstico' (unidade de apoio à diagnose e terapia, farmácia, laboratório central de saúde pública), 'hospital com ambulatório' (hospital especializado, hospital geral), 'pronto atendimento' (pronto atendimento, pronto-socorro geral) e 'outros' (centro de parto normal - isolado, hospital/ dia - isolado, secretaria de saúde, unidade de vigilância em saúde, unidade mista, unidade móvel de nível pré-hospitalar na área de urgência).

Dessa forma, os serviços de saúde estudados possuem estruturas distintas, com algumas tipicamente ambulatoriais, como as unidades básicas, e outras menos usuais neste tipo de análise, como os hospitais especializados. A categorização segundo o tipo de estabelecimento buscou identificar se os procedimentos ambulatoriais cotidianos concentram-se nas unidades particularmente responsáveis por essa modalidade de atenção, levando em consideração que a reforma do sistema de saúde local orientou-se para a expansão desses serviços, principalmente aqueles relacionados à ESF.

De posse dessas informações, foi possível, então, descrever e analisar os dados a partir da distribuição dos serviços segundo as variáveis: 'esfera administrativa' e 'natureza organizacional', a fim de se identificar evidências sobre o comando do município sobre os serviços ambulatoriais e, consequentemente, a sua capacidade de gestão sobre a APS.

A variável 'esfera administrativa' faz referência ao ente federado sob o qual o serviço é administrado: 'Municipal'; 'Estadual'; 'Federal'; e 'Privada'. A análise da esfera administrativa privada refere-se aos serviços privados contratados ou conveniados que integram o SUS, todos sob gestão municipal e com financiamento público.

A variável 'natureza organizacional' agrega diferentes formas de organização dos serviços de saúde conforme as categorias: 'Administração direta da saúde'; 'Administração direta de outros órgãos'; 'Administração indireta'; 'Empresa privada'; e 'Sem fins lucrativos'.

Os indicadores da utilização foram baseados em dados secundários disponíveis no SIA-SUS sobre os procedimentos selecionados dentro dos seus subgrupos, de acordo com a Tabela de Procedimentos, Medicamentos, 
Órteses e Próteses e Materiais Especiais do SUS: i. Diagnóstico em laboratório clínico (análise de caracteres físicos, elementos e sedimentos na urina); ii. Diagnóstico por ultrassonografia (todos), Diagnóstico por tomografia (todos), Diagnóstico por ressonância magnética (todos); iii. Métodos diagnósticos em especialidades (eletrocardiograma); e iv. Consultas/Atendimentos/ Acompanhamentos (todos).

Esses indicadores foram adaptados como traçadores dos exames laboratoriais básicos; exames com diagnóstico por imagem; métodos gráficos; e das consultas médicas, dimensões aqui entendidas como estruturantes do manejo do paciente no âmbito ambulatorial.

O uso de traçadores tem como premissa que alguns problemas específicos de saúde podem ser utilizados para a análise da prestação de serviços a partir da interação entre os prestadores, usuários e o ambiente. Entre as principais características desse método, destacam-se: a existência de marcadores pragmáticos que levem em conta o sistema de informação existente e a capacidade potencial da rede de prestação de serviços de saúde, e o estabelecimento de um mínimo do que se espera do sistema de atenção à saúde (KESSNER; KALK; SINGER, 1973; KESSNER; KALK; SINGER, 1992).

O procedimento análise de caracteres físicos, elementos e sedimentos na urina, conhecido usualmente como EAS, foi selecionado como exemplo de um dos itens de menor complexidade e de grande frequência dentro do subgrupo Diagnóstico em laboratório clínico. Entende-se que esse exame é o mínimo necessário e esperado da oferta ambulatorial dos sistemas de saúde, e, assim, funcionou como traçador dos exames laboratoriais básicos.

Todos os procedimentos dos subgrupos Diagnóstico por ultrassonografia, Diagnóstico por tomografia e Diagnóstico por ressonância magnética foram analisados conjuntamente como traçadores dos exames com diagnóstico por imagem e refletem alguns dos itens de maior complexidade, custo e exigência de equipamentos para a sua realização dentro de uma rotina diagnóstica comandada pelo ambulatório, ou seja, sem a necessidade de instalações mais complexas.

O procedimento eletrocardiograma foi eleito dentro do subgrupo Métodos diagnósticos em especialidades, enquanto traçador dos métodos gráficos, por ser altamente relevante para o monitoramento das doenças cardiovasculares, uma das condições clínicas mais comuns no ambulatório.

Todos os procedimentos do subgrupo Consultas/Atendimentos/Acompanhamentos foram analisados, e o seu conjunto utilizado como traçador das consultas médicas, por serem as atividades das quais se espera serem as mais prevalentes e relevantes no ambulatório médico, além das mais completas (conceitualmente, compreendem a anamnese, o exame físico e a elaboração de hipóteses ou conclusões diagnósticas, solicitação de exames complementares, quando necessários, e prescrição terapêutica).

Foi utilizado o software $\mathrm{SAS}^{\circledR}$ (Statistical Analysis System) para a análise do total e distribuição percentual dos procedimentos realizados segundo o tipo de classificação do estabelecimento e as variáveis selecionadas.

Testes de hipótese e o controle de variáveis para fins de maior comparabilidade não pareceram vantajosos, uma vez que a composição dos grupos é bastante heterogênea pelas próprias características do sistema local de saúde. Sendo assim, os resultados encontrados foram apresentados em frequências absolutas e relativas e foram considerados relevantes para esses conjuntos.

\section{Resultados e discussão}

De um total de 384 serviços de saúde, 03 foram excluídos por não possuírem cadastro no CNES e, portanto, informações que permitissem suas análises, totalizando, assim, um universo de 381 serviços ambulatoriais. 
O grupo 'outros' agregou apenas 08 serviços de saúde. A sua análise foi pouco relevante no estudo e não considerada nos resultados.

As tabelas 1, 2, 3 e 4 apresentam, segundo esfera administrativa, natureza organizacional e tipo de estabelecimento dos serviços de saúde, o total e a distribuição percentual dos procedimentos ambulatoriais específicos adotados como traçadores na análise da utilização: análise de caracteres físicos, elementos e sedimentos na urina (EAS); exames com diagnóstico por imagem (ultrassonografia/tomografia/ressonância magnética); eletrocardiograma; e consultas/atendimentos/acompanhamentos; respectivamente.

Tabela 1. Serviços de saúde ( $n=381$ ) segundo tipo de classificação do estabelecimento; total e distribuição percentual de procedimentos realizados (análise de caracteres físicos, elementos e sedimentos na urina) no município do Rio de Janeiro, 2012

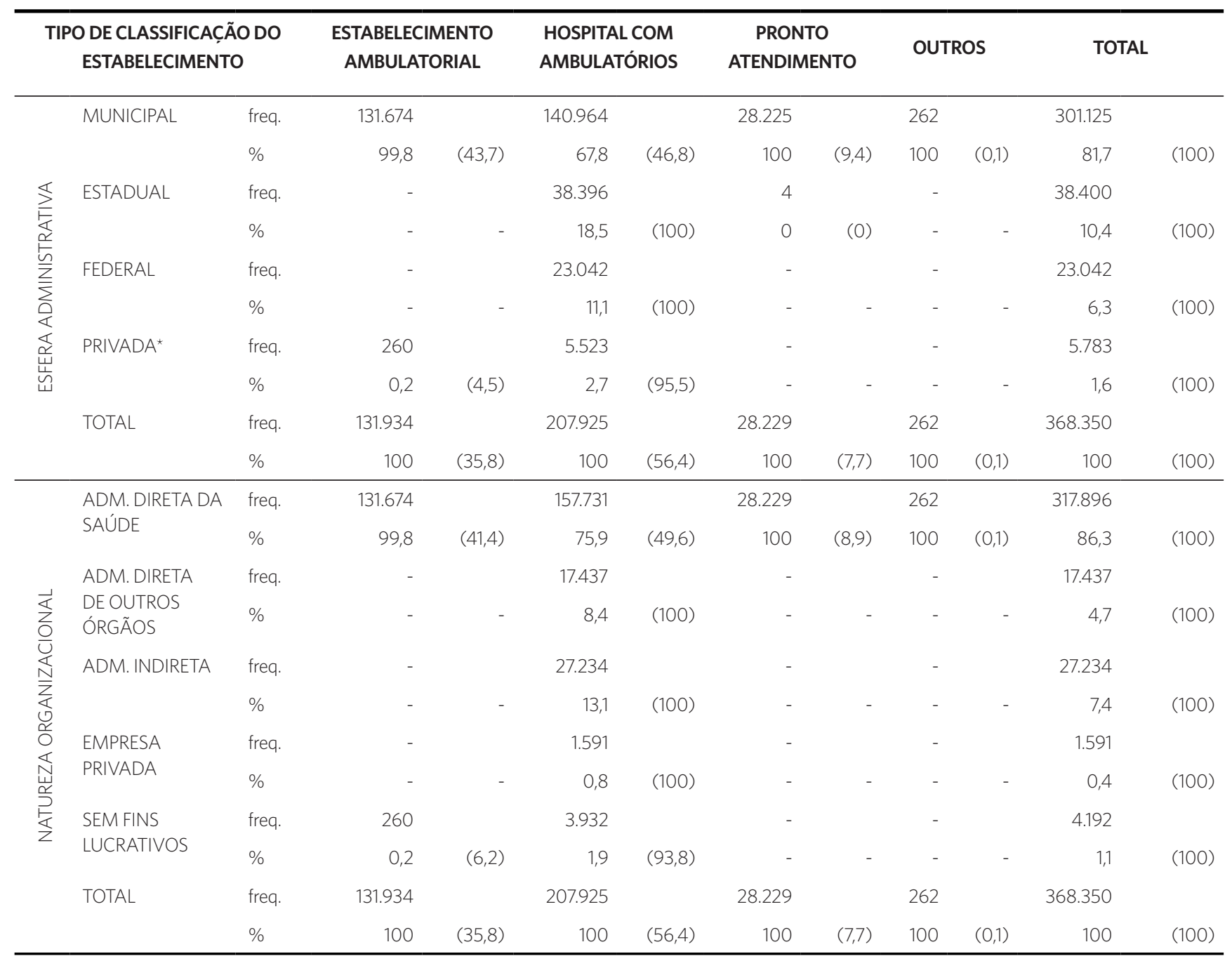

Fonte: Elaboração própria, a partir do CNES/MS e do SIA-SUS

* Serviços privados contratados ou conveniados que integram o SUS. Todos sob gestão municipal. 
Tabela 2. Serviços de saúde ( $n=381$ ) segundo tipo de classificação do estabelecimento; total e distribuição percentual de procedimentos realizados (exames com diagnóstico por imagem - ultrassonografia/tomografia/ressonância magnética) no município do Rio de Janeiro, 2012

\begin{tabular}{|c|c|c|c|c|c|c|c|c|c|c|c|c|c|c|}
\hline \multicolumn{3}{|c|}{$\begin{array}{l}\text { TIPO DE CLASSIFICAC̣ÃO } \\
\text { DO ESTABELECIMENTO }\end{array}$} & \multicolumn{2}{|c|}{$\begin{array}{l}\text { ESTABELECIMENTO } \\
\text { AMBULATORIAL }\end{array}$} & \multicolumn{2}{|c|}{$\begin{array}{c}\text { SERVIC̣O } \\
\text { DE APOIO } \\
\text { DIAGNÓSTICO }\end{array}$} & \multicolumn{2}{|c|}{$\begin{array}{l}\text { HOSPITAL COM } \\
\text { AMBULATÓRIOS }\end{array}$} & \multicolumn{2}{|c|}{$\begin{array}{c}\text { PRONTO } \\
\text { ATENDIMENTO }\end{array}$} & \multicolumn{2}{|c|}{ OUTROS } & \multicolumn{2}{|c|}{ TOTAL } \\
\hline \multirow{10}{*}{ 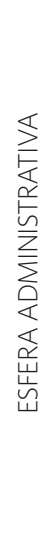 } & \multirow[t]{2}{*}{ MUNICIPAL } & freq. & 74.877 & & - & & 75.490 & & - & & - & & 150.367 & \\
\hline & & $\%$ & 92,8 & $(49,8)$ & - & - & 44,3 & $(50,2)$ & - & - & - & - & 56,2 & (100) \\
\hline & \multirow[t]{2}{*}{ ESTADUAL } & freq. & - & & - & & 36.934 & & 966 & & - & & 37.900 & \\
\hline & & $\%$ & - & - & - & - & 21,7 & $(97,5)$ & 100 & $(2,5)$ & - & - & 14,2 & (100) \\
\hline & \multirow[t]{2}{*}{ FEDERAL } & freq. & - & & - & & 50.622 & & - & & - & & 50.622 & \\
\hline & & $\%$ & - & - & - & - & 29,7 & (100) & - & - & - & - & 18,9 & (100) \\
\hline & \multirow[t]{2}{*}{ PRIVADA* } & freq. & 5.786 & & 13.738 & & 7.341 & & - & & 2.028 & & 28.893 & \\
\hline & & $\%$ & 7,2 & (20) & 100 & $(47,5)$ & 4,3 & $(25,4)$ & - & - & 100 & (7) & 10,8 & (100) \\
\hline & \multirow[t]{2}{*}{ TOTAL } & freq. & 80.663 & & 13.738 & & 170.387 & & 966 & & 2.028 & & 267.782 & \\
\hline & & $\%$ & 100 & $(30,1)$ & 100 & $(5,1)$ & 100 & $(63,6)$ & 100 & $(0,4)$ & 100 & $(0,8)$ & 100 & (100) \\
\hline \multirow{12}{*}{ 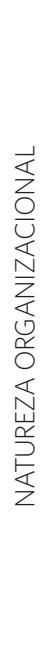 } & \multirow{2}{*}{$\begin{array}{l}\text { ADM. DIRETA } \\
\text { DA SAÚDE }\end{array}$} & freq. & 74.877 & & - & & 121.886 & & 966 & & - & & 197.729 & \\
\hline & & $\%$ & 92,8 & $(37,9)$ & - & - & 71,5 & $(61,6)$ & 100 & $(0,5)$ & - & - & 73,8 & (100) \\
\hline & \multirow{2}{*}{$\begin{array}{l}\text { ADM. DIRETA } \\
\text { DE OUTROS } \\
\text { ÓRGÃOS }\end{array}$} & freq. & - & & - & & 19.645 & & - & & - & & 19.645 & \\
\hline & & $\%$ & - & - & - & - & 11,5 & (100) & - & - & - & - & 7,3 & (100) \\
\hline & \multirow{2}{*}{$\begin{array}{l}\text { ADM. } \\
\text { INDIRETA }\end{array}$} & freq. & - & & - & & 21.515 & & - & & - & & 21.515 & \\
\hline & & $\%$ & - & - & - & - & 12,6 & (100) & - & - & 0 & - & 8 & (100) \\
\hline & \multirow{2}{*}{$\begin{array}{l}\text { EMPRESA } \\
\text { PRIVADA }\end{array}$} & freq. & 92 & & 13.315 & & - & & - & & 1.788 & & 15.195 & \\
\hline & & $\%$ & 0,1 & $(0,6)$ & 96,9 & $(87,6)$ & - & - & - & - & 88,2 & $(11,8)$ & 5,7 & (100) \\
\hline & \multirow{2}{*}{$\begin{array}{l}\text { SEM FINS } \\
\text { LUCRATIVOS }\end{array}$} & freq. & 5.694 & & 423 & & 7.341 & & - & & 240 & & 13.698 & \\
\hline & & $\%$ & 7,1 & $(41,6)$ & 3,1 & $(3,1)$ & 4,3 & $(53,6)$ & - & - & 11,8 & $(1,8)$ & 5,1 & (100) \\
\hline & \multirow[t]{2}{*}{ TOTAL } & freq. & 80.663 & & 13.738 & & 170.387 & & 966 & & 2.028 & & 267.782 & \\
\hline & & $\%$ & 100 & $(30,1)$ & 0 & $(5,1)$ & 100 & $(63,6)$ & 100 & $(0,4)$ & 100 & $(0,8)$ & 100 & (100) \\
\hline
\end{tabular}

Fonte: Elaboração própria, a partir do CNES/MS e do SIA-SUS.

* Serviços privados contratados ou conveniados que integram o SUS. Todos sob gestão municipal. 
Tabela 3. Serviços de saúde ( $n=381$ ) segundo tipo de classificação do estabelecimento; total e distribuição percentual de procedimentos realizados (eletrocardiograma) no município do Rio de Janeiro, 2012

\begin{tabular}{|c|c|c|c|c|c|c|c|c|c|c|c|c|c|c|}
\hline \multicolumn{3}{|c|}{$\begin{array}{l}\text { TIPO DE CLASSIFICAC̣ÃO } \\
\text { DO ESTABELECIMENTO }\end{array}$} & \multicolumn{2}{|c|}{$\begin{array}{l}\text { ESTABELECIMENTO } \\
\text { AMBULATORIAL }\end{array}$} & \multicolumn{2}{|c|}{$\begin{array}{c}\text { SERVIC̣O } \\
\text { DE APOIO } \\
\text { DIAGNÓSTICO }\end{array}$} & \multicolumn{2}{|c|}{$\begin{array}{l}\text { HOSPITAL COM } \\
\text { AMBULATÓRIOS }\end{array}$} & \multicolumn{2}{|c|}{$\begin{array}{c}\text { PRONTO } \\
\text { ATENDIMENTO }\end{array}$} & \multicolumn{2}{|c|}{ OUTROS } & \multicolumn{2}{|c|}{ TOTAL } \\
\hline \multirow{5}{*}{ 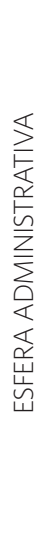 } & \multirow[t]{2}{*}{ MUNICIPAL } & freq. & 63.485 & & - & & 25.180 & & 29.524 & & 93 & & 118.282 & \\
\hline & & $\%$ & 99,3 & $(53,7)$ & - & - & 43,5 & $(21,3)$ & 100 & (25) & 100 & $(0,1)$ & 70,3 & $(100)$ \\
\hline & \multirow[t]{2}{*}{ FEDERAL } & freq. & - & & - & & 9.648 & & - & & - & & 9.648 & \\
\hline & & $\%$ & - & - & - & - & 16,7 & $(100)$ & - & - & - & - & 5,7 & (100) \\
\hline & PRIVADA* & freq. & 443 & & 16.753 & & 2.226 & & - & & - & & 19.422 & \\
\hline \multirow{7}{*}{ 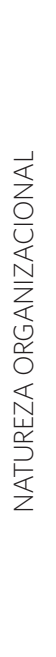 } & \multirow{2}{*}{$\begin{array}{l}\text { ADM. DIRETA } \\
\text { DA SAÚDE }\end{array}$} & $n$ & 63.496 & & - & & 42.953 & & 29.524 & & 93 & & 136.066 & \\
\hline & & $\%$ & 99,3 & $(46,7)$ & - & - & 74,3 & $(31,6)$ & 100 & $(21,7)$ & 100 & $(0,1)$ & 80,9 & $(100)$ \\
\hline & \multirow{2}{*}{$\begin{array}{l}\text { ADM. DIRETA } \\
\text { DE OUTROS } \\
\text { ÓRGÃOS }\end{array}$} & $n$ & - & & - & & 5.878 & & - & & - & & 5.878 & \\
\hline & & $\%$ & - & - & - & - & 10,2 & $(100)$ & - & - & - & - & 3,5 & (100) \\
\hline & \multirow{2}{*}{$\begin{array}{l}\text { ADM. } \\
\text { INDIRETA }\end{array}$} & $\mathrm{n}$ & - & & - & & 6.784 & & - & & - & & 6.784 & \\
\hline & & $\%$ & - & - & - & - & 11,7 & $(100)$ & - & - & - & - & 4 & (100) \\
\hline & $\begin{array}{l}\text { EMPRESA } \\
\text { PRIVADA }\end{array}$ & $n$ & - & & 16.753 & & - & & - & & - & & 16.753 & \\
\hline
\end{tabular}

Fonte: Elaboração própria, a partir do CNES/MS e do SIA-SUS.

* Serviços privados contratados ou conveniados que integram o SUS. Todos sob gestão municipal. 
Tabela 4. Serviços de saúde ( $n=381$ ) segundo tipo de classificação do estabelecimento; total e distribuição percentual de procedimentos realizados (consultas/atendimentos/acompanhamentos) no município do Rio de Janeiro, 2012

\begin{tabular}{|c|c|c|c|c|c|c|c|c|c|c|c|c|}
\hline \multicolumn{3}{|c|}{$\begin{array}{l}\text { TIPO DE CLASSIFICAC̣ÃO } \\
\text { DO ESTABELECIMENTO }\end{array}$} & \multicolumn{2}{|c|}{$\begin{array}{l}\text { ESTABELECIMENTO } \\
\text { AMBULATORIAL }\end{array}$} & \multicolumn{2}{|c|}{$\begin{array}{l}\text { HOSPITAL COM } \\
\text { AMBULATÓRIOS }\end{array}$} & \multicolumn{2}{|c|}{$\begin{array}{c}\text { PRONTO } \\
\text { ATENDIMENTO }\end{array}$} & \multicolumn{2}{|c|}{ OUTROS } & \multicolumn{2}{|c|}{ TOTAL } \\
\hline \multirow{10}{*}{ 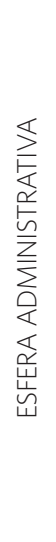 } & MUNICIPAL & freq. & 13.532 .839 & & 2.175 .107 & & 3.121 .240 & & 78.268 & & 18.907 .454 & \\
\hline & & $\%$ & 97 & $(71,6)$ & 51,9 & $(11,5)$ & 97,8 & $(16,5)$ & 74,2 & $(0,4)$ & 88,2 & $(100)$ \\
\hline & ESTADUAL & freq. & 160.388 & & 906.320 & & 69.170 & & 13.777 & & 1.149 .655 & \\
\hline & & $\%$ & 1,1 & (14) & 21,6 & $(78,8)$ & 2,2 & (6) & 13,1 & $(1,2)$ & 5,4 & $(100)$ \\
\hline & FEDERAL & freq. & 7.700 & & 852.378 & & - & & - & & 860.078 & \\
\hline & & $\%$ & 0,1 & $(0,9)$ & 20,3 & $(99,1)$ & - & - & - & - & 4 & (100) \\
\hline & PRIVADA* & freq. & 248.755 & & 260.109 & & - & & 13.392 & & 522.256 & \\
\hline & & $\%$ & 1,8 & $(47,6)$ & 6,2 & $(49,8)$ & - & - & 12,7 & $(2,6)$ & 2,4 & (100) \\
\hline & TOTAL & freq. & 13.949 .682 & & 4.193 .914 & & 3.190 .410 & & 105.437 & & 21.439 .443 & \\
\hline & & $\%$ & 100 & $(65,1)$ & 100 & $(19,6)$ & 100 & $(14,9)$ & 100 & $(0,5)$ & 100 & (100) \\
\hline \multirow{12}{*}{ 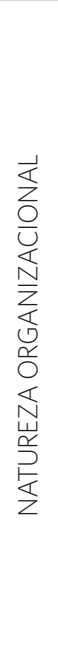 } & ADM. DIRETA & $n$ & 13.693 .227 & & 3.112 .421 & & 3.190 .410 & & 78.268 & & 20.074 .326 & \\
\hline & DA SAÚDE & $\%$ & 98,2 & $(68,2)$ & 74,2 & $(15,5)$ & 100 & $(15,9)$ & 74,2 & $(0,4)$ & 93,6 & (100) \\
\hline & ADM. DIRETA & $\mathrm{n}$ & - & & 427.801 & & - & & 13.777 & & 441.578 & \\
\hline & $\begin{array}{l}\text { DE OUTROS } \\
\text { ÓRGÃOS }\end{array}$ & $\%$ & - & - & 10,2 & $(96,9)$ & - & - & 13,1 & $(3,1)$ & 2,1 & (100) \\
\hline & ADM. & $\mathrm{n}$ & 7.700 & & 393.583 & & - & & - & & 401.283 & \\
\hline & INDIRETA & $\%$ & 0,1 & $(1,9)$ & 9,4 & $(98,1)$ & - & - & - & - & 1,9 & (100) \\
\hline & EMPRESA & $\mathrm{n}$ & 36.303 & & 8.133 & & - & & 6.014 & & 50.450 & \\
\hline & PRIVADA & $\%$ & 0,3 & (72) & 0,2 & $(16,1)$ & - & - & 5,7 & $(11,9)$ & 0,2 & (100) \\
\hline & SEM FINS & $n$ & 212.452 & & 251.976 & & - & & 7.378 & & 471.806 & \\
\hline & LUCRATIVOS & $\%$ & 1,5 & (45) & 6 & $(53,4)$ & - & - & 7 & $(1,6)$ & 2,2 & (100) \\
\hline & TOTAL & $\mathrm{n}$ & 13.949 .682 & & 4.193 .914 & & 3.190 .410 & & 105.437 & & 21.439 .443 & \\
\hline & & $\%$ & 100 & $(65,1)$ & 100 & $(19,6)$ & 100 & $(14,9)$ & 100 & $(0,5)$ & 100 & (100) \\
\hline
\end{tabular}

Fonte: Elaboração própria, a partir do CNES/MS e do SIA-SUS.

* Serviços privados contratados ou conveniados que integram o SUS. Todos sob gestão municipal. 
A categoria 'hospital com ambulatórios' realizou mais da metade dos exames de EAS $(56,4 \%)$ e dos exames com diagnóstico por imagem (63,6\%). Nota-se, assim, que os estabelecimentos ambulatoriais não são os principais em utilização de procedimentos adotados como proxy daqueles mais frequentes e básicos, como também dos menos comuns e mais complexos.

Da mesma forma, o setor hospitalar sedia exames que poderiam estar concentrados em centros de apoio especializados. De fato, de acordo com o CNES, o município não conta com nenhum estabelecimento exclusivo de apoio diagnóstico. Essa configuração é anômala à lógica do mercado, que, por uma questão de eficiência de escala, concentra os seus procedimentos de apoio diagnóstico em grandes centros especializados, a exemplo dos centros de diagnóstico por imagem e dos laboratórios centrais.

Assim, sugere-se que o grupo 'estabelecimento ambulatorial', que deve funcionar como linha de frente entre os serviços de APS, não está retendo a maior demanda por exames, independentemente da complexidade e da frequência com que são rotineiramente utilizados no manejo dos pacientes ambulatoriais.

Entende-se, assim, que os exames complementares de diagnóstico, fundamentais na oferta integral da atenção primária, ainda que providos sob comando da administração municipal, estão pulverizados, sem eficiência econômica, em vários serviços próprios.

Por outro lado, como era de se esperar, a categoria 'estabelecimento ambulatorial' foi a que realizou a maior quantidade de consultas/atendimentos/acompanhamentos $(65,1 \%)$. Inesperado, entretanto, é o fato desse grupo de serviços dividir com o grupo hospitalar a realização da maioria dos eletrocardiogramas, ultrapassando-o inclusive em alguns pontos percentuais: $38,0 \%$ contra $34,4 \%$, respectivamente.

Os serviços do tipo 'pronto atendimento' ocuparam a terceira posição na distribuição percentual de todos os procedimentos realizados que foram avaliados como traçadores, com exceção dos exames com diagnóstico por imagem, para os quais ficaram em último, com uma produção residual de $0,4 \%$.

Os serviços de apoio diagnóstico realizaram $10 \%$ dos eletrocardiogramas, apenas $5,1 \%$ dos exames com diagnóstico por imagem e, curiosamente, não registraram a realização de EAS. Os resultados, nesse caso, são ainda mais anômalos ao se considerar que esse grupo inclui o Laboratório Central de Saúde Pública (Lacen), serviço administrado pela Secretaria Estadual de Saúde. Os serviços de apoio diagnóstico também não realizaram consultas/atendimentos/ acompanhamentos.

Analisando internamente os dois grupos que mais se destacaram, ou seja, os estabelecimentos ambulatoriais e os hospitais com ambulatórios, todos os procedimentos tratados como traçadores concentraram-se na esfera municipal.

Esse mesmo comportamento é observado na distribuição total entre as esferas, com destaque para os exames de EAS e consultas/atendimentos/acompanhamentos, procedimentos que totalizam na administração municipal $81,7 \%$ e $88,2 \%$ da produção, respectivamente. Ainda que em menor proporção, os estabelecimentos municipais também predominam na provisão de exames com diagnóstico por imagem $(56,2 \%)$ e de eletrocardiogramas (70,3\%), aqui utilizados como proxy de procedimentos mais complexos e de maior valor.

Esses dados sugerem que o município pode estar chamando para si os procedimentos de maior complexidade, na tentativa de resolver problemas de coordenação da atenção, e, assim, absorvendo dentro dele uma parte que comumente seria referenciada para outros entes federados. Nesse sentido, especula-se a premissa de que possa haver um déficit de coordenação no Rio de Janeiro. As partes, assim, não estariam sob regulação única e gestão unificada. 
O município pode estar realizando procedimentos de maior complexidade por 'path dependence', ou seja, herdou um modelo de rede de atenção que já era, tradicionalmente, no passado, estruturado para ofertar serviços de maior complexidade; ou por 'feedback positivo', ou seja, a existência de eventos em momentos críticos fez com que serviços de média complexidade fossem agregados pela esfera municipal na ausência da possibilidade de delegá-los a outras esferas federativas.

É questionável a condição em que a maioria dos serviços de APS, e que respondem pela engrenagem principal na reorientação do modelo de atenção à saúde - haja vista que engloba as clínicas da família -, não seja protagonista na oferta de procedimentos considerados como o mínimo que um sistema de saúde deve prover. Neste caso específico, os estabelecimentos ambulatoriais, que representam mais de $70 \%$ da rede de serviços, respondem por somente $35,8 \%$ de exames tidos como um dos procedimentos laboratoriais mais comuns (EAS) e, portanto, contam com o apoio da rede ambulatorial dos hospitais que, com $18,4 \%$ dos serviços, realizam $56,4 \%$ desses procedimentos.

Esse modelo, assim sendo, não se alinha à experiência internacional, que mostra que há uma expectativa de maior eficiência e redução de custos ao tirar o paciente do ambiente hospitalar, que deve se tornar especializado somente em emergências, traumas e cirurgias eletivas.

Dessa forma, para a garantia da oferta integral da assistência à saúde no município, é necessário que esses diferentes serviços se articulem em rede, garantindo que os usuários tenham acesso aos procedimentos que estão ausentes dos serviços que configuram, de acordo com os normativos do Ministério e da SMS, a 'porta de entrada' do sistema.

Ainda assim, fica evidente o esforço individual do município na provisão e no financiamento de um sistema de atenção primária integral, que conta pouco com o amparo de outros entes e, em decorrência disso, busca oferecer sozinho um conjunto amplo de ações e serviços de saúde, para além das suas competências, na integração de uma rede regionalizada e hierarquizada.

Quanto à natureza organizacional, analisando internamente os dois grupos que mais se destacaram na realização desses procedimentos, ou seja, os estabelecimentos ambulatoriais e os hospitais com ambulatórios, todos os traçadores, além de concentrarem-se na esfera municipal, foram majoritariamente realizados sob o comando da administração direta da saúde.

Esse mesmo comportamento é observado na distribuição total entre as esferas, com destaque para as consultas/atendimentos/ acompanhamentos, que totalizam $93,6 \%$ da sua produção na administração direta da saúde.

Os dados reforçam, assim, a hipótese de que a maior parte da produção dos serviços esteja fortemente sob comando municipal, haja vista que, além de ser característica desta esfera, está sob administração direta da saúde.

Ganhou destaque o fato de os serviços de saúde, sabidamente administrados por OS mediante a celebração de Contrato de Gestão, estarem cadastrados na base do CNES como 'Administração direta da saúde', ainda que as OS pertençam a um grupo formado exclusivamente por pessoas jurídicas de direito privado sem fins lucrativos.

\section{Considerações finais}

Com a reorganização do sistema de saúde brasileiro e o princípio da descentralização e municipalização dos serviços de saúde, o modelo centralizado, cujas origens são anteriores aos anos 1930, vem sendo substituído por um sistema no qual os municípios devem assumir a gestão da APS.

A recente reforma do setor saúde do município do Rio de Janeiro, a partir de 2009, com priorização da APS, acompanhada de 
um modelo inovador de relação contratual no gerenciamento dos serviços de saúde, reforça a relevância da escolha do Rio de Janeiro como exemplo de reorientação de modelo.

A literatura acumulada, bem como a própria natureza das suas definições conceituais, impõem convergência entre a atenção ambulatorial e a APS. Este trabalho considerou coerente contar com a suposição inicial de que a reforma da APS no município do Rio de Janeiro teve impacto direto sobre atenção ambulatorial, incluindo as dimensões aqui analisadas quanto à capacidade de governo e adequação de modelo.

Nesse sentido, foi oportuno adotar traçadores da utilização de serviços ambulatoriais rotineiros na oferta do cuidado integral e usuais nos protocolos internacionais voltados à garantia da resolubilidade do cuidado em saúde na APS.

Análises de demanda usualmente são do tipo ecológico de séries temporais. Todavia, este estudo é seccional para o ano de 2012 e fez uso de bases massivas secundárias para testar modelos de análise para um futuro estudo em série. A demanda, que, usualmente, na econometria, é uma variável independente, foi tratada por aproximação como uma repercussão da quantidade de procedimentos realizados. Ao não analisar uma série histórica, esta pesquisa foi incapaz de mensurar tendências de aumento ou diminuição da utilização ao longo do tempo ou no período pré e pós-reforma no município. Todavia, é um desenho que permite comparações com parâmetros usualmente utilizados pela literatura acadêmica e pelas esferas de gestão do serviço público de saúde.

As análises dos serviços ambulatoriais segundo esfera administrativa e natureza organizacional constataram o predomínio dos serviços e dos respectivos procedimentos realizados na esfera administrativa municipal e na administração direta da saúde, evidenciando, assim, forte comando do município e, consequentemente, capacidade de gestão sobre a APS. Esses resultados convergem para o modelo racional esperado de organização dos sistemas locais de saúde, onde tais serviços devem estar sob responsabilidade local.

Os dados apresentados neste artigo sugerem efeitos não intencionais da política de saúde, onde a ênfase na universalização da APS reforçou a atuação do município na atenção ambulatorial e, por conseguinte, na especialização de funções federativas na direção dos preceitos da Reforma Sanitária e do SUS, embora não seja um objetivo nominado da reforma em curso no Rio de Janeiro.

Por outro lado, há indicativos de que houve um processo de sobrecarga orçamentária local, com concentração excessiva no município de procedimentos aqui utilizados como proxy daqueles mais complexos e de maior valor, os quais, segundo o modelo preconizado, deveriam ser cofinanciados por outros entes federativos.

Não foram estudadas, especificamente, as relações intergovernamentais que compõem a gestão do sistema de saúde local, porém, os dados sugerem que o município do Rio de Janeiro integralizou e internalizou em sua rede os diferentes serviços ambulatoriais, incluindo os de maior complexidade, possivelmente por dificuldades de coordenação e articulação com as esferas estadual e federal.

A constatação do predomínio municipal sobre o conjunto dos serviços ambulatoriais direciona o desafio do município para a articulação entre os diferentes tipos de estabelecimentos que compõem a sua rede de atenção à saúde. Ainda que estejam concentrados sob comando da administração municipal, o que atenua as dificuldades de provisão, que dependem de relações intergovernamentais, procedimentos considerados vitais na rotina de prestação de cuidados ambulatoriais podem estar em grande parte fora dos serviços considerados a 'linha de frente' da APS, como as unidades básicas de saúde tradicionais e as clínicas da família.

Em contexto latino-americano, no qual, 
historicamente, coexistem diferentes visões de APS, recente consenso entre especialistas confere à coordenação entre os distintos níveis assistenciais o papel de componente essencial de uma APS abrangente (ALMEIDA; GIOVANELLA; NUNAN, 2012; HAGGERTY; YAVICH; BÁSCOLO, 2009).

Por fim, ainda que o caso estudado repercuta para o conjunto do debate sobre a organização da APS e a própria estruturação do SUS, este artigo não cessa a necessidade de aprofundamento das temáticas abordadas. No caso dos sistemas federais, como o Brasil, em que vigora uma soberania compartilhada, constitui-se pauta especial a necessidade de aprofundamento de processos negociados de decisões e responsabilidades no setor saúde.

A maioria dos municípios brasileiros não tem arrecadação própria expressiva, e a sua condição limitada de autonomia política e financeira gera dependência das transferências intergovernamentais de recursos (PRADO; QUADROS; CAVALCANTI, 2003; LIMA, 2007; LIMA, 2013) e limita o exercício dos governos locais (SOUZA, 2002).

Ainda que o Rio de Janeiro represente um exemplo potencial de organização de sistemas locais de saúde, possui uma estrutura orçamentária atípica frente aos demais municípios brasileiros, de forma que coloca em dúvida se outros sistemas locais dariam conta de tamanha intensidade de investimento setorial na ausência de forte apoio intergovernamental.

\section{Colaboradores}

G. Z. Portela e J. M. Ribeiro participaram da concepção geral, análise de dados, discussão dos resultados e redação final do artigo.

\section{Referências}

\footnotetext{
ALMEIDA, P. F.; GIOVANELLA, L.; NUNAN, B. A. Coordenação dos cuidados em saúde pela atenção primária à saúde e suas implicações para a satisfação dos usuários. Saúde em Debate, Rio de Janeiro, v. 36, n. 94, p. 375-391, 2012.
}

BERMAN, P. Organization of ambulatory care provision: a critical determinant of health system performance in developing countries. Bulletin of the World Health Organization, Genebra, v. 78, n. 6, p. 791$802,2000$.

CUETO, M. The origins of primary health care and selective primary health care. American Journal of
Public Health, Nova York, v. 94, n. 11, p. 1864-1874, 2004.

GIOVANELLA, L. A atenção primária à saúde nos países da União Européia: configurações e reformas organizacionais na década de 1990. Cadernos de Saúde Pública, Rio de Janeiro, v. 22, n. 5, p. 951-963, 2006.

Atenção Primária à Saúde seletiva ou abrangente? Cadernos de Saúde Pública, Rio de Janeiro, v. 24, supl. 1, p. s21-s23, 2008.

Redes integradas, programas de gestão clínica e generalista coordenador: análise das reformas recentes 
do setor ambulatorial na Alemanha. Ciência \&t Saúde

Coletiva, Rio de Janeiro, v. 16, supl.1, p. 1081-1096, 2011.

HAGGERTY, J. L.; YAVICH, N.; BÁSCOLO, E. P. Grupo

de Consenso sobre un Marco de Evaluación de la Atención Primaria en América Latina. Un marco de evaluación de la atención primaria de salud en América Latina. Revista Panamericana de Salud Pública, v. 26, n. 5, p. 377-384, 2009.

KESSNER, D. M.; KALK, C. E.; SINGER, J. A. Assessing health quality -the case for tracers. New England Journal of Medicine, Boston, v. 288, n. 4, p. 189-194, 1973.

Evaluación de la calidad de la salud por el método de los procesos trazadores. In: WHITE, K.

L. (Org.). Investigaciones sobre servicios de salud: una antología. Washington, DC: Organización Panamericana de la Salud, 1992. p. 555-563.

LIMA, L. D. Federalismo, relações fiscais e financiamento do Sistema Único de Saúde: distribuição de receitas vinculadas à saúde nos orçamentos municipais e estaduais. Rio de Janeiro: Museu da República, 2007.

A coordenação federativa do sistema público de saúde no Brasil. In: FUNDAÇÃO OSWALDO CRUZ. A saúde no Brasil em 2030 - prospecção estratégica do sistema de saúde brasileiro: organização e gestão do sistema de saúde. Rio de Janeiro: Fiocruz; Ipea; Ministério da Saúde; Secretaria de Assuntos Estratégicos da Presidência da República, 2013. p. 73-139.

ORGANIZATION FOR ECONOMIC COOPERATION AND DEVELOPMENT (OECD); WORLD HEALTH ORGANIZATION (WHO); STATISTICAL OFFICE OF THE EUROPEAN UNION (EUROSTAT). A System of Health Accounts, 2011 edition. Paris: OECD Publishing, 2011. Disponível em: <http://www.who.int/healthaccounts/methodology/sha2011.pdf $>$. Acesso em: 27 mar. 2016.
PORTELA, G. Z. Avaliação do desempenho da atenção primária à saúde no município do Rio de Janeiro. 2013. 151 f. Tese (Doutorado em Saúde Pública) - Escola Nacional de Saúde Pública Sergio Arouca, Fundação Oswaldo Cruz, Rio de Janeiro, 2013.

PRADO, S.; QUADROS, W.; CAVALCANTI, C. E. (Org.) Partilha de recursos na federação brasileira. São Paulo: FAPESP; FUNDAP; Brasília, DF: IPEA, 2003.

RIBEIRO, J. M.; ALCOFORADO, F. Mecanismos de governança e o desenho institucional da Secretaria de Saúde do Município do Rio de Janeiro (RJ). Ciência $\mathcal{E} t$ Saúde Coletiva, Rio de Janeiro, v. 21, n. 5, p. 1339-1349, 2016.

SOUZA, C. Governos e sociedades locais em contextos de desigualdades e de descentralização. Ciência \&t Saúde Coletiva, Rio de Janeiro, v. 7, n. 3, p. 431-442, 2002.

STARFIELD, B. Atenção primária: equilíbrio entre necessidades de saúde, serviços e tecnologia. Brasília, DF: UNESCO; MS, 2002.

TRAVASSOS, C.; MARTINS, M. Uma revisão sobre os conceitos de acesso e utilização de serviços de saúde. Cadernos de Saúde Pública, Rio de Janeiro, v. 20, supl. 2, p. S190-S198, 2004.

WORLD HEALTH ORGANIZATION (WHO). Health care systems in transition. Production template and questionnaire. Copenhague: WHO Regional Office for Europe, 1996. Disponível em: <http://www.euro.who. int/_data/assets/pdf_file/0019/120277/E72450.pdf>. Acesso em: 27 mar. 2016.

\footnotetext{
Recebido para publicação em abril de 2016

Versão final em novembro de 2016

Conflito de interesses: inexistente

Suporte financeiro: não houve
} 